\title{
OAW
}

Österreichische Akademie

der Wissenschaften

TA'10 - Call for Papers

\section{Dezember 2009}

Cyberscience 1.2

Nanotagungen in

Wien, Seattle \&

Kuala Lumpur 



\section{Editorial}

\section{Werte Leserinnen und Leser!}

Ein wichtiger Meilenstein des Grundlagenprojekts der langiährigen ITA-Mitarbeiterin, Dr. Mahshid Sotoudeh, zur technischen Ausbildung unter Nachhaltigkeitsgesichtspunkten* war die Organisation der internationalen Konferenz "Engineering Education in a Sustainable Development (EESD)" im September 2008 in Graz; im vergangenen Frühjahr erschien schließlich ihr Buch mit dem Titel "Technical Education for sustainability. An analysis of needs in the $21^{\text {st }}$ century" (siehe dazu mehr auf S. 18). Das Projekt kam dieser Tage zu einem krönenden Abschluss: Dr. Sotoudeh absolvierte an der Fakultät für Verfahrenstechnik der Technischen Universität Graz auf Basis dieses Themas erfolgreich ihr Habilitationskolloquium und wird die Venia Legendi im Fach "Technikfolgenabschätzung und Nachhaltigkeit" erhalten. Sie wird damit nach unserem Wissensstand die einzige Frau sein, die im Fach TA habilitiert ist und die zweite in Österreich überhaupt. Dies ist eine verdiente Anerkennung für ihren unermüdlichen Einsatz in diesem Fachbereich - wir gratulieren sehr herzlich! Zugleich handelt es sich aber auch um ein willkommenes Zeichen dafür, dass TA - wie zuvor bereits in Deutschland - auch in Österreich akademisch "angekommen" ist.

Aus den vielen weiteren Beiträgen zu diesem Newsletter möchte ich besonders den Call for Papers für die nächstiährige TA-Konferenz in Wien hervorheben (siehe S. 5). Am 31. Mai und 1. Juni 2010 werden wir das Thema "Ethisierung der Technik" und ihre Bedeutung für die TA ins Zentrum rücken. Die TA'10 wird übrigens die zehnte ihrer Art sein. Wir freuen uns über das anhaltende, auch internationale Interesse an dieser Tagung und hoffen auf viele spannende Einreichungen und Ihre Mitwirkung an den Debatten auf der Konferenz selbst!

Ich wünsche Ihnen viel Freude beim Lesen unserer Berichte zu ITA-Projekten und der Neuigkeiten rund um die TA sowie angenehme Festtage!

* Siehe dazu bereits ITA-News März 2007, S. 6, März 2008, S. 10, und Dez. 2008, S. 12-13.

Michael Nentwich

\section{Inhalt}

\section{ITA-Projekte}

Cyberscience 1.2: Das Web 2.0

und die Zukunft der Wissenschaft

EMAS auch für Forschungseinrichtungen ..........3

Der Klimagipfel der WeltbürgerInnen

$\mathrm{TA}^{\prime}$ 10: Die Ethisierung der Technik -

und ihre Bedeutung für die

Technikfolgenabschätzung

\section{TA-aktuell}

Integration von Wissenschaft und

Gesellschaft in der Forschung.....

ITA goes public - Die Lange Nacht der

Forschung 2009

Fünf Jahre Netzwerk

Technikfolgenabschätzung (NTA).

Mögliche Auswirkungen auf die

Gesundheit durch künstlich hergestellte

Nanomaterialien.

Entzauberung der Nanotechnologie -

Bericht von der ersten Konferenz der

"Society for the Study of Nanoscience

and Emerging Technologies"

NANOtech Malaysia 2009 und

Nanotechnologie-Forum in Kuala Lumpur......11

Aktuelle Publikationen

ITA-Veranstaltungen

Kontakt

Impressum 


\section{Cyberscience 1.2: Das Web 2.0 und die Zukunft der Wissenschaft}

\author{
Werden Wikipedia, Second Live, Blogs, \\ Twitter, Facebook \& Co die Wissenschafts- \\ kommunikation revolutionieren? Ein ak- \\ tuelles ITA-Workingpaper' ${ }^{1}$ gibt erste Ant- \\ worten auf die Frage, welchen Einfluss \\ das sogenannte "Soziale Web" oder \\ "Web 2.0" auf die Wissenschaft haben \\ könnte.
}

Die ITA-Studie "Cyberscience" (2003) ${ }^{2}$ untersuchte den Einfluss von E-Mail, Online-Datenbanken, Groupware, Hypertext und E-Journals auf die Wissenschaft und kam zu dem Ergebnis, dass alle Bereiche der Wissenschaft betroffen sind und die Konsequenzen auch qualitativer Natur sind. 2003 steckte das sogenannte Web 2.0 noch in den Kinderschuhen, während es heute allgegenwärtig ist: Hunderttausende weltweit, darunter auch viele Wissenschafterlnnen, sind Teil des enorm wachsenden „Sozialen Netzes" geworden, das die neven Dienste fördern.

Ein paar Beispiele für typische Web 2.0-Anwendungen: Auf speziellen Plattformen entstehen neuartige soziale Netzwerke, die bottom-up entstehen. Internetnutzerlnnen, die gemeinsame Interessen teilen, erhalten auf besonders einfache Weise die Möglichkeit, sich selbst im Netz darzustellen und mit anderen zu vernetzen. Es gibt bereits spezialisierte Netzwerkdienste für WissenschafterInnen. Andere Web 2.0-Applikationen ermöglichen es den Nutzerlnnen, selbst und unkompliziert zu Autorlnnen zu werden, insbesondere die Web-Tagebücher ("Weblogs", "Blogs"). Immer mehr Wissenschafterlnnen schreiben regelmäßig solche öffentlichen Artikel. Auch Microblogging-Dienste, also Dienste zum Versenden von sehr kurzen Meldungen (z. B. Twitter), werden von etlichen Wissenschafterlnnen in ihrer täglichen Praxis zum Informationsaustausch genutzt. Auch alle Formen von Wikis, also kollaborative und im Gegensatz zu den früheren Groupware-Anwendungen öffentliche Schreibumgebungen, haben in der Wissenschaft teilweise Fuß gefasst. Das bekannteste Beispiel ist Wikipedia, die auch von Forscherlnnen täglich aktiv und passiv genutzt wird. ${ }^{3}$ Ebenfalls dem Teilen von Wissen dient die thematische Sammlung von Links zu Webseiten und
Online-Publikationen durch „social bookmarking". Dadurch entstehen die sogenannten Folksonomien, die im neuen Netz die herkömmlichen, von Spezialistlnnen gewarteten Taxonomien ergänzen. Schließlich werden virtuelle Welten durch ihre Nutzerlnnen und deren Onlineverhalten gestaltet und geprägt; in Second Life etwa findet sich auch wissenschaftlicher Inhalt. ${ }^{4}$

Während 2009 kein Zweifel bestehen kann, dass Cyberscience (1.0) längst Realität ist und in vielen Bereichen die traditionellen Weisen, Wissenschaft zu betreiben, abgelöst hat, stellt sich in Hinblick auf die neuen Dienste des Web 2.0 erneut die Frage, wie viel davon für die Wissenschaft funktional sein und sich in der täglichen Praxis durchsetzen wird. Die vermutlich häufigste erste Reaktion, die man von Wissenschafterlnnen erhält, ist mangelnde Nutzenerwartung. Mit zunehmender Vertrautheit mit den neven Diensten relativiert sich diese Einschätzung zwar bisweilen, die Frage nach dem Mehrwert ist freilich berechtigt. In der ITA-Untersuchung werden u. a. folgende Themen behandelt: Zeitmangel, die Gefahr des Informationsüberlastung, das fehlende Anreizsystem, die Frage der inhaltlichen Qualität und die Konkurrenz zwischen Forscherlnnen. Die Antworten auf die Frage, ob sich das Web 2.0 in der Wissenschaft durchsetzen könnte, führen dann zur weiteren, noch spannenderen Frage, was das für die Wissenschaft bedeuten würde. Diskutiert werden etwa ein Wandel des Qualitätssicherungssystems, das Verschwimmen der Grenze zwischen interner und externer Wissenschaftskommunikation, die schleichende Integration von Publikations- und Konversationsmedien sowie Fragen des Privatsphärenschutzes.

Sieht man sich dies unter dem Blickwinkel der bisherigen Cyberscience-Folgenabschätzung an, erkennt man, dass es sich um keine wirklich neuen Themen handelt. Die unter dem Titel Web 2.0 bekannt gewordenen Dienste, die sich mittlerweile auch mit einer gewissen Dynamik in der Scientific Community verbreiten, verstärken möglicherweise Trends, die im Internetzeitalter der Wissenschaft angelegt sind. Die Tools sind unbestreitbar besser geworden, um weiterhin die ursprünglichen Ziele zu erreichen. 
Dennoch kann man die Web 2.0-Applikationen technisch und organisatorisch von ihren Vorgängern unterscheiden: In gewisser Weise ist das Internet auch in Hinblick auf seine Nutzung durch die Wissenschaft "gereift". Im Softwarejargon gesprochen, ist somit durch das Web 2.0 eher ein "Update auf Cyberscience 1.2" als eine neue „Release Cyberscience 2.0" zu erwarten.
1 Nentwich, M., 2009, Cyberscience 2.0 oder 1.2? Das Web 2.0 und die Zukunft der Wissenschaft. ITA manu:scripts, Nr. ITA-09-02, epub.oeaw.ac.at/ita/ita-manuscript/ita_09_02.pdf.

2 Mittlerweile vollständig online und frei zugänglich hier: hw.oeaw.ac.at/3188-7.

3 Vgl. dazu schon in ITA-News März 2009, S. 7.

4 Vgl. dazu schon in ITA-News Dez. 2008, S. 2.

Michael Nentwich

\section{EMAS auch für Forschungseinrichtungen}

\begin{abstract}
Das freiwillige Öko-Audit der Europäischen Union - EMAS - richtet sich seit 1993 an Unternehmen, die zur nachhaltigen Entwicklung beitragen wollen. Das ITA hat erstmalig in einem Projekt ausgelotet, inwiefern sich das Verfahren auch auf Forschungseinrichtungen anwenden lässt und hat dabei sehr gute Erfahrungen gemacht.
\end{abstract}

EMAS ist die Kurzbezeichnung für "Eco Management and Audit Scheme", auch bekannt als EU-Öko-Audit. EMAS fußt auf einer EU-Verordnung aus 1993 zum Umweltmanagement, das ein freiwilliges Instrument für eine nachhaltige Entwicklung in Unternehmen sein soll. ${ }^{1}$ Will sich ein Unternehmen EMAS-zertifizieren lassen, so wird ein umfassender interner Prozess initiiert, der alle Verfahren und Produkte eines Unternehmens umfasst. Ein eigens gebildetes Umweltteam und interne Umweltauditorlnnen durchleuchten den Betrieb, um Potenziale für umweltfreundlichere Abläufen auszuloten. Es werden Input/Output-Analysen durchgeführt, eine Umweltpolitik formuliert sowie detaillierte und überprüfbare Maßnahmenkataloge erstellt. Ähnlich wie bei der ISO-9000-Zertifizierung werden im anschließenden externen Audit auch standardisierte Verfahrensabläufe evaluiert. Eine Evaluierung von EMAS im Auftrag der Generaldirektion Umwelt im Rahmen des Projektes „EVER" zeigte 2005, dass EMAS von den Unternehmen nicht nur als ein Instrument des Umweltmanagements, sondern auch als Beitrag zu einer umfassenderen Organisationskultur und als ein wichtiger Imagefaktor bezeichnet wird.

Das österreichische Umweltministerium und der Österreichische Wasser- und Abfallwirtschaftsverband (ÖWAV) als Kooperationspart- ner haben das Projekt „Mit EMAS zu ökologisch aktiven Gemeinden, Verbänden und öffentlichen Einrichtungen" initiiert, um EMAS bekannt zu machen und dessen Einführung und Verbreitung mit Hilfe von Workshops zu erleichtern. ${ }^{2}$ Zwei Mitarbeiterlnnen des ITA haben sich 2008/ 2009 im Rahmen dieses Projekts zu internen "Umweltauditorlnnen" ausbilden lassen. Zugleich wurde ein Umweltmanagementprozess am ITA in Gang gesetzt, der noch nicht abgeschlossen ist. Das dreiköpfige "Umweltteam" und das zweiköpfige "Auditorlnnenteam" haben eine Reihe von Maßnahmen identifiziert, die bereits in mehreren internen Workshops vorgestellt und diskutiert wurden. Einige dieser Maßnahmen befinden sich bereits in der Umsetzungsphase.

Die Herausforderung für eine Forschungseinrichtung war es, die auf Unternehmen und öffentliche Einrichtungen (wie z. B. Gemeinden oder Abfallwirtschaftsverbände) zugeschnittenen Verfahren auf die Bedürfnisse und Besonderheiten einer wissenschaftlichen Einrichtung zu übersetzen. Hauptthemen waren neben Stromund Papierverbrauch sowie der ökologischen Beschaffung und Abfallentsorgung vor allem die in einem international vernetzten, in vielen EU-Forschungsprojekten kooperierenden Institut unvermeidliche Reisetätigkeit. Wir werden an dieser Stelle und in einem Forschungsbericht über Erfahrungen bei der Umsetzung berichten.

\footnotetext{
1 Die aktuell gültige Fassung ist die Verordnung (EG) Nr. 761/2001 vom 19.03.2001. Eine Novellierung ist in Arbeit und ist noch 2009 geplant.

2 Das Programm für die neve Workshopsreihe für 2010 zum Download:

www.emas.gv.at/article/articleview/72885/1/16773/.
}

Mahshid Sotoudeh, Michael Nentwich 


\section{Der Klimagipfel der WeltbürgerInnen}

\begin{abstract}
Am 26. September haben in Wien fast 100 ÖsterreicherInnen zeitgleich mit 4.400 TeilnehmerInnen aus 38 Ländern über global relevante, klimapolitische Themen diskutiert und Empfehlungen für politische Entscheidungsträgerlnnen ausgearbeitet. Diese werden bei der 15 . UN-Klimakonferenz (COP 15, 7.-18.12.09 in Kopenhagen) an die Delegierten übergeben.
\end{abstract}

Wie bereits in unserem Newsletter im Juni 2009 berichtet, ist das ITA als Partner am internationalen Projekt "World Wide Views on Global Warming" (WWViews) beteiligt, das vom Dänischen Technologierat (DBT) koordiniert wird. Im März dieses Jahres begann das Projektteam gemeinsam mit einem internationalen wissenschaftlichen Beirat mit der Ausarbeitung eines Fragenkatalogs zu fünf ausgewählten, für COP 15 relevante Bereiche zum Thema Klimawandel: Definition von Klimazielen, Strategien und Maßnahmen gegen den Klimawandel, Anpassungsstrategien an den Klimawandel und technische Forschung, Entwicklung und Innovation Finanzierung der Maßnahmen und Innovationen.

Dieser Fragenkatalog war Basis für den Diskussions- und Empfehlungsprozess rund um die Strategien im weltweiten Kampf gegen die globale Erwärmung beim "Klimagipfel der WeltbürgerInnen" am 26. September in Wien. Zeitgleich zum österreichischen Klimagipfel (innerhalb von 36 Stunden) fanden weltweit weitere 44 methodisch idente Bürgerlnnenkonferenzen statt.

Am Ende der Veranstaltung in Wien hatten die Teilnehmerlnnen ihre Meinung zu den Fragen individuell abgegeben und gemeinsam $17 \mathrm{Emp}$ fehlungen formuliert. Alle Ergebnisse wurden schon während des Prozesses auf eine Internetplattform gestellt und so für alle Interessierten und anderen Teilnehmerlnnen auf der ganzen Welt sichtbar gemacht.

Die Ergebnisse zeigen deutlich, dass den Teilnehmerlnnen das Thema Klimawandel sehr wichtig ist und sie sich von den PolitikerInnen ein klares Signal erwarten. Dies betrifft ein verbindliches Klimaabkommen mit strengen Reduktionszielen, deren Nichteinhaltung auch geahndet wird.

\section{Die drei dringlichsten Empfehlungen der ÖsterreicherInnen lauten:}

- Die notwendigen Klimarichtlinien müssen weltweit verbindlich umgesetzt werden. Diese können durch Technologietransfer sowie Energie- und Emissionsnormen das globale Problem der Treibhausemissionen im Rahmen der nationalen Möglichkeiten jedes Landes schnell und nachhaltig lösen.

- Die Forschung zu kosteneffizienten, $\mathrm{CO}_{2}$-armen Technologien soll forciert und der weltweite Technologietransfer unterstützt werden.

- Ein uneingeschränkter Zugang zu Bildung und Information ist die Voraussetzung für eine effektive Teilnahme am Klimaentscheidungsprozess, in den die tatsächlich Betroffenen des Klimawandels miteingebunden werden müssen.

Am 13. November wurden die Ergebnisse und damit auch die Empfehlungen der österreichischen Bürgerlnnen vom WWViews-Team des ITA an den stv. Leiter der österreichischen Delegation in Kopenhagen, Sektionschef DI Günter Liebel (Abt. Allgemeine Umweltpolitik, Lebensministerium), übergeben.

Die wichtigsten Anliegen aller Bürgerlnnen weltweit wurden von den dänischen Initiatoren von WWViews in Form eines Policy reports (neun Empfehlungen an die Politik) am 19. November dem dänischen Parlament übergeben. Am 10. Dezember sollen die Ergebnisse und Empfehlungen des "Klimagipfels der Weltbürgerlnnen" im Rahmen der Konferenz in Kopenhagen präsentiert werden.

Alle Ergebnisse des Klimagipfels der Weltbürgerlnnen: results.wwviews.org/.

Informationen zu WWViews Österreich: www.oeaw.ac.at/ita/wwviews.

Informationen zu WWViews International: www.wwviews.org.

Medienplattform mit Videos, Fotos, Audiointerviews: teknologiraad.surfoffice.eu/.

Sabine Stemberger, Ulrike Bechtold 


\section{TA'10: Die Ethisierung der Technik - und ihre Bedeutung für die Technikfolgenabschätzung}

\begin{abstract}
Am 31. Mai und 1. Juni 2010 wird die zehnte internationale TA-Konferenz in Wien stattfinden und sich der sogenannten „Ethisierung" der Technikdebatten und deren Bedeutung für die Technikfolgenabschätzung widmen. Als KeynoteSpeaker konnten Wolfgang van den Daele und Nicole Karafyllis gewonnen werden. Es folgt der Call for Papers.
\end{abstract}

In Debatten um Technik und Ethik geht es zumeist um moralische Fragen, wie etwa darum, ob wir dürfen, was technisch machbar ist. Dies ist traditionell das Terrain der akademischen Ethik. In aktuellen Technikkontroversen sind jedoch ethische Fragen längst zum Politikum geworden.

Auf der TA'10 soll daher das Thema Technik und Ethik in einen größeren Zusammenhang gestellt werden. Denn mit dem Bedeutungszuwachs des ethischen Diskurses für die Problematisierung und Gestaltung von Technologien wandeln sich die Ansprüche an Technology Governance. Verhandlungs- und Beteiligungsformen ändern sich, bestehende Technologien erscheinen in nevem Licht, neve Technologien nehmen ethische Vorgaben auf. Bisher implizite Wertbezüge werden explizit. "Ethisierung" wird damit zu einem wichtigen Faktor und zugleich Indikator für ein verändertes Regulieren und Gestalten von Technologien im 21. Jahrhundert.

Obwohl die Begriffe Technik und Ethik - in einem Atemzug genannt - dazu einladen: Die $\mathrm{TA}^{\prime} 10$ sollte sich nicht in Disputen über die Leistungsfähigkeit einer Ethik der Technik erschöpfen. Vielmehr sind darüber hinaus gehende Diskussionen erwünscht, insbesondere zu folgenden Themenstellungen:

- Ethik und Governance: Wie ändern sich Konflikt-, Politik- und Legitimationsmuster, wenn Technologien aus ethischer Perspektive thematisiert werden - und nicht mehr unter den Aspekten von Risiko und ökonomischem Nutzen?
- TA und Ethik: Ändern sich Aufgaben und Methoden der TA im Kontext der "Ethisierung"? In welches (Konkurrenz-)Verhältnis gerät TA zu ethikspezifischer Expertise (z. B. ELSI-Forschung, nationale Ethikräte)?

- Ethik der Technik: Was sind tragfähige normative Prinzipien einer Regulierung technischen Handelns? Bezogen auf TA: Was sind die normativen Grundlage der TA? Braucht sie welche?

- Partizipation: Wenn es um Wertfragen geht, kann man Laien nicht ausschließen. Welchen Stellenwert hat partizipative Technikbewertung mit Laienbeteiligung heute?

- Normative Prinzipien der Technikgestaltung wie Nachhaltigkeit, Vorsorgeprinzip oder Schutz der Privatsphäre werden gerade nicht unter dem Titel der Ethik eingeführt. Welchen Einfluss haben sie auf die Verhandlung und Gestaltung von Technologien?

- "Technologien der Ethik" (in Anlehnung an Foucault): Ob erweiterte Beteiligung durch EGovernance oder Klimaschutz durch lowcarbon technologies: Viele Technologien sind heute eng mit Gemeinwohlansprüchen verbunden. Gleichzeitig zeichnen sie Bilder aktiver, informierter BürgerInnen oder disziplinierter EnergiesparerInnen. Welche Normierungs- und Kontrolleffekte haben "gute" Technologien?

- Technik ohne Ethik: In manchen Technologien kommt die "Ethisierung" nicht zum Tragen. Welche normativen Voraussetzungen liegen dieser "Ethikabstinenz" zugrunde?

Das ITA lädt zur Einreichung von Beiträgen ein, die sich den im Call genannten Fragen widmen. Die Einreichungen sollen auf 1-2 Seiten Forschungsthema und Fragestellung skizzieren sowie die methodische Vorgangsweise und wichtige Resultate darlegen. Einreichungen bitte bis 29.1.2010 per Email an:

tamail@oeaw.ac.at.

Konferenzhomepage: www.oeaw.ac.at/ita/ta10.

Für das ITA: Alexander Bogner 


\section{Integration von Wissenschaft und Gesellschaft in der Forschung}

\begin{abstract}
Vom 19. bis 21. November hielt das Netzwerk für transdisziplinäre Forschung tdnet seine internationale Fachtagung an der Universität Bern ab. Im Zentrum stand dieses Jahr das Thema der Integration - auf theoretischer, methodologischer, institutioneller und praktischer Ebene. Während einer Vielzahl von Präsentationen wurden grundsätzliche Fragen inter- und transdisziplinärer Forschung erörtert.
\end{abstract}

Das Label der Transdisziplinarität meint nicht immer exakt das Gleiche, das wurde auch während dieser Tagung wieder deutlich. Transdisziplinarität kann auf Forschung jenseits der Disziplinengrenzen ebenso hinweisen wie auf Forschung, die über die engen Grenzen des Wissenschaftsbetriebs hinausgeht, gesellschaftliche Problemstellungen in den Blick nimmt und gesellschaftliche Akteure und Betroffenengruppen in den Forschungsprozess integriert.

Es wird aber auch nicht überall mit dem Transdisziplinaritäts-Label versehen, was einer solchen Charakterisierung standhält. So sehr sich der Begriff in Kontinentaleuropa etablieren konnte, so groß ist die Vielfalt an alternativen Bezeichnungen im internationalen Vergleich. Julie Thompson Klein (Wayne State University) führte dafür in ihrem Plenarvortrag einige Beispiele an, weitere folgten in anderen Beiträgen. Hierzu zählen Formulierungen wie „problem-orientierte" oder "Iösungs-orientierte Forschung", "Integrative studies", "Integration and Implementation Sciences", "Interventionsforschung" oder auch das nevere Label der "Science of Team Science".

Eine Podiumsdiskussion widmete sich dem Zusammenhang zwischen feministischer Forschung, Genderforschung und Transdisziplinarität. Der wissenschaftshistorische Rückblick rief in Erinnerung, wie sehr die Entwicklung transdisziplinärer Wissenschaft durch erstere beide Forschungsrichtungen begründet, motiviert und geprägt ist. Zugleich wurde Gender kaum als Kategorie oder Thema in den einzelnen Projektpräsentationen angesprochen.

Vor dem Hintergrund der Fülle, Diversität und mitunter auch Widersprüchlichkeit an Forschung mit transdisziplinären Qualitäten, verwundert es nicht, dass während der Tagung Wünsche laut wurden, transdisziplinäre Forschung klarer von anderer Forschung abzugrenzen und die interne Qualitätssicherung weiterzuentwickeln. Dieser Wunsch spiegelt sich auch in neuen Handbüchern zu inter- und transdisziplinärer Forschung wieder. Dennoch fand die Forderung, transdisziplinäre Forschung streng nach Kriterien und Mechanismen disziplinärer Wissenschaft zu entwickeln, keine breite Zustimmung.

Offen wurde darüber diskutiert, ob sich unter Transdisziplinarität eher ein Prinzip, ein Ansatz, eine Vorgehensweise oder eine Methode verstehen lasse. In vielen der Beiträge wurde darüber hinaus thematisiert, was es bedeutet und welche besonderen Vorkehrungen es braucht, wenn die Schwelle zwischen Wissenschaft und Gesellschaft (bzw. Politik) während des Forschungsprozesses gezielt überschritten wird. Die breit gefächerten Praxisbeispiele halfen, das Ausmaß dieser Fragestellung zu überblicken, konnten aber nur in beschränktem Ausmaß übergreifende Antworten bereitstellen. Einige Beiträge widmeten sich wiederum primär der Praxisreflexion und methodologischen, epistemologischen und ontologischen Fragen, wie etwa jener von Ulrike Felt (Universität Wien) zu "epistemic living spaces". Dieses Konzept verdeutlicht, dass konkrete Lebensräume oder Kontexte bestimmte Formen der Wissenserzeugung vorwegnehmen und andere ausschließen.

Insgesamt waren beide Anteile vertreten - die Praxis transdisziplinärer Forschung (etwa im Rahmen humanökologisch ausgerichteter Projekte der Entwicklungszusammenarbeit) ebenso wie ihre kritische Reflexion. Die Integration beider Seiten stellte sich als herausfordernde Aufgabe dar. Auch standen sich eine Reflexion "von innen", die in den Forschungsprozess eingebettet ist, und eine Reflexion "von außen", wie jene, die die Wissenschaftsforschung anbietet, gegenüber. Ausreichend offene Fragen also für eine Tagung 2010!

Die Kurzfassungen der Beiträge finden Sie unter: www.transdisciplinarity.ch/e/Conference/ international/2009/index.php.

Karen Kastenhofer 


\section{ITA goes public - Die Lange Nacht der Forschung 2009}

\begin{abstract}
Am 7. November 2009 fand in ganz Österreich die Lange Nacht der Forschung (LNF) statt, bei der sich universitäre und außeruniversitäre Forschungseinrichtungen einer breiten Öffentlichkeit vorstellen konnten. Von Sonnenuntergang um 16:27 bis Mitternacht stand das ITATeam in der Aula der Wissenschaften bereit, um die BesucherInnen über die wissenschaftliche Arbeit am Institut zu informieren, Fragen zu beantworten und über mögliche Auswirkungen neuer Technologien zu diskutieren.
\end{abstract}

Schon im Vorfeld der Veranstaltung zeigte sich, dass es 2009 weit mehr Veranstaltungsorte und Stationen geben würde als all die Jahre zuvor. Alleine in Wien gab es hever 157 Stationen, österreichweit waren es 570. In Wien wurden 146.880 Besucherlnnen gezählt, insgesamt waren es 366.000! Das zeigt deutlich, dass immer mehr Forschungsinstitutionen in Österreich den direkten Kontakt zur Bevölkerung suchen und diese das Angebot mit Begeisterung annimmt.

Die Lange Nacht der Forschung wurde in der Österreichischen Akademie der Wissenschaften in Wien durch Wissenschaftsminister Hahn gemeinsam mit ÖAW-Präsident Denk eröffnet. Im Anschluss besuchten BM Hahn und ÖAWPräsidiumsmitglieder die Stationen der ÖAWForschungseinrichtungen. Am ITA-Stand ließen sie sich über den "Klimagipfel der WeltbürgerInnen" informieren, der Teil des bislang größten weltweiten Bürgerlnnenbeteilungsverfahrens im Rahmen des ITA-Projekts "WWViews on Global Warming" war.

Schwerpunkt der diesjährigen Präsentationen des ITA waren zwei Projekte, bei denen die Partizipation von Bürgerlnnen im Mittelpunkt stand: Das Projekt "CIVISTI - Visionen von Bürgerlnnen zu Wissenschaft, Technologie und Innovation" stellte die Ergebnisse von mehreren Workshops in sieben Ländern vor, bei denen die Beteiligten Visionen entwickelten, die von einem wünschenswerten Umgang mit älteren Menschen bis hin zu "Beamen" reichten. Die Besucherlnnen hatten an der ITA-Station die Mög- lichkeit, eigene Visionen zu formulieren. Diese zusätzlich gesammelten Visionen werden in das Projekt CIVISTI einfließen. Das rege Interesse am Projekt selbst und an den Visionen hat deutlich gezeigt, dass BürgerInnen motiviert und gewillt sind, einen Beitrag zur Gestaltung einer "nachhaltigen Zukunft" zu leisten, umso mehr, als sie selbst von den Auswirkungen neuer Technologien und Innovationen betroffen sind bzw. sein werden.

Viel Interesse zeigten die BesucherInnen auch für das Projekt "WWViews on Global Warming", in dessen Rahmen im Vorfeld der 15. UN-Klimakonferenz (COP 15) im Dezember d. J. in Kopenhagen weltweit 4.400 BürgerInnen zu klimarelevanten Themen diskutiert und Empfehlungen ausgearbeitet haben (siehe auch S. 4). Die Besucherlnnen konnten am Laptop die Ergebnisse aller beteiligten Nationen aufrufen und miteinander vergleichen und/oder sich an Pinwänden die Meinungen von Expertlnnen, politischer EntscheidungsträgerInnen etc. in Form von Statements ansehen. Hauptattraktion vor allem für Kinder und Jugendliche waren vier Filme, die beim Klimagipfel der Weltbürgerlnnen als Input gedient hatten.

Viele Besucherlnnen interessierten sich für den Bereich Technikfolgenabschätzung (TA) an sich. "Was ist TA überhaupt? Worüber forscht das ITA? Was sind die Themen der TA?", waren nur einige der Einstiegsfragen in spannende Diskussion rund um das Thema TA.

Es war für das ITA-Team wieder eine spannende Herausforderung, sich den zahlreichen und unterschiedlichsten Fragen der sehr heterogen zusammengesetzten Interessierten zu stellen. Sehr spezifische Fragen zu Beteiligungsverfahren oder zu den Ergebnissen von WWViews wechselten mit eher allgemeinen Fragen zu Nanotechnologien und diversen anderen ITAForschungsthemen ab.

Aufgrund der unüblichen Herangehensweise und den ungewohnten Fragestellungen der interessierten Besucherlnnen wurde das ITA-Team mit durchaus ungewohnten Ansichten und Perspektiven konfrontiert, was von den Forscherln- 
nen übereinstimmend als "sehr bereichernd" empfunden wurde.

Das ITA möchte mit seiner regelmäßigen Teilnahme an der LNF einen Beitrag dazu leisten, interessierte Bürgerlnnen über die Ergebnisse seiner wissenschaftlichen Arbeit zu informieren und mit diesem Dialog die Beziehung und das Verständnis zwischen Wissenschaft und Gesellschaft verbessern.

Petra Wöchter

\section{Fünf Jahre Netzwerk Technikfolgenabschätzung (NTA)}

Am 11. und 12. November fand das 5. Jahrestreffen des Netzwerks Technikfolgenabschätzung in Berlin statt. Es wurden neve Tendenzen der TA international diskutiert, sowie die Zukunft des Netzwerks und seine Fortsetzung beschlossen.

Dem Anlass entsprechend wurde das Jahrestreffen des Netzwerks der deutschsprachigen TA-Community im Sitzungssaal des Ausschusses für Technikfolgenabschätzung des Deutschen Bundestags abgehalten. Das NTA wurde vor fünf Jahren gegründet und besteht aus Wissenschafterlnnen und Institutionen mit unterschiedlichsten Arbeitsgebieten und Ausrichtungen der TA. Mittlerweile umfasst das Netzwerk 206 persönliche Mitglieder und 41 Institutionen. Über die zugehörige E-Mail-Liste werden derzeit 366 Wissenschafterlnnen erreicht.

Das Jahrestreffen wurde von Armin Grunwald mit einem Bericht über zwei Tagungen zur TA ${ }^{1}$ eingeleitet. Anschließend diskutierten die etwa 40 Teilnehmerlnnen über neve Tendenzen und Entwicklungen in der TA und in TA-nahen Politikbereichen. So wurden u. a. folgende Tendenzen beobachtet:

- Hinwendung zu frühen Stadien der Technikentwicklung

- Zunahme der Beratung zu Forschungsprogrammen

- Steigende Nachfrage nach TA, die sich den großen Herausforderungen (Klimawandel, Globalisierung, Ressourcenverbrauch) widmet

- Vermehrte Berücksichtigung von Governance-Aspekten.

Aus den USA wurde berichtet, dass es zwar konkrete Überlegungen zur Wiedereinrichtung des OTA (Office of Technology Assessment) gibt, dass die an der Session ${ }^{2}$ beteiligten Kongressabgeordneten jedoch keine genaue Vorstellung über den Zweck einer solchen Einrichtung hätten. Seit einigen Jahren übernimmt das GAO (General Accounting Office), der US-Rechnungshof, TA-Aufgaben.

Ein weiterer Schwerpunkt des Jahrestreffens war die Diskussion der Ergebnisse einer Mitgliederumfrage zum bisherigen Funktionieren und zur Zukunft des Netzwerks selbst. Diese Umfrage wurde schon bei der Gründung der NTA vor fünf Jahren geplant und hever im Juli und August vom Koordinationsteam anlässlich des fünfjährigen Bestehens des NTA durchgeführt. Zu Beginn stand die Frage im Mittelpunkt, ob es für das Netzwerk einer genaueren Definition für TA bedarf, um auf die Frage, welche Mitglieder und Institutionen zu dem Netzwerk gehören sollen, präzise beantworten zu können. In der Diskussion kristallisierte sich heraus, dass der schlanke Organisationsgrad, der auf Basis der Eigeninitiative der Beteiligten beruht, am besten zu der äußerst heterogenen Forschungs- bzw. Beratungsaktivitäten der TA passt. In der Mitgliederbefragung hatten sich $97 \%$ der TeilnehmerInnen für die Weiterführung des Netzwerkes ausgesprochen. Auch wenn viele Ziele, die sich das NTA gesetzt hatte, bisher nicht erreicht wurden, sprach sich die überwiegende Mehrzahl dafür aus, diese Ziele auch weiterhin zu verfolgen. Die Umfrage ergab weiters den Wunsch nach einer Verstärkung der Kommunikation nach innen wie nach außen sowie einer stärkeren Interaktion und Kooperation zwischen den Mitgliedern. Positiv aufgenommen wurde der Vorschlag, statt der "starrer" thematischer Arbeitsgruppen in Zukunft eher Workshops zu organisieren. Schließlich wurde das Netzwerk einvernehmlich um weite- 
re fünf Jahre verlängert und dem Koordinationsteam der Auftrag erteilt, die kreative Fortentwicklung des Netzwerks zu betreiben.

Am Vormittag des Folgetages lud die Arbeitsgruppe "Informations- und Kommunikationstechnologien (luK)" des NTA zu einer öffentlichen Sitzung ein. Thema der Sitzung waren die Entwicklungen neuer Dienste des Internets (Stichwort: Web 2.0) und die Frage, ob diese für die TA-Community nutzbar gemacht werden können. Zu Beginn der Sitzung präsentierte Michael Nentwich in einem Überblicksvortrag ${ }^{3}$ Internetdienste für Wissenschafterlnnen, die ähnlich wie Facebook thematische Diskussionsforen, Abstimmungsmechanismen und darüber hinaus Suchmöglichkeiten in wissenschaftlicher Literatur anbieten. In der anschließenden Diskussion wurden die Vorteile (komfortable Unterstützung der Kommunikation und Vernetzung) und Nachteile („Verzettelung" in weiteren Kommunikationskanälen) diskutiert. Letzt- lich kann der potenzielle Gewinn für das Netzwerk aber nur anhand von eigenen Erfahrungen bestimmt werden. Die Arbeitsgruppe luK hat in diesem Sinne mit "ResearchGATE" einen Selbstversuch gestartet.

Weitere Informationen zum NTA sind zu finden unter: www.netzwerk-ta.net.

\footnotetext{
GWTF-Jahrestagung 2008, Berlin, 21.-22.1 1.08, „Technology Assessment Reloaded? Konzepte, Methoden, Erfahrungen"; vgl. ITA-News Dez. 2008, S. 8; und Session "The Renaissance of Technology Assessment" im Rahmen der Jahreskonferenz 2009 der Society for Social Studies of Science (4S), 28.-31.10.09 in Washington.

2 "S\&T Advice for Congress: The Campaign to Restore the Office of Technology Assessment", convention3.allacademic.com/one/ssss/4s09/index.php?click key=1\&cmd $=$ Multi+Search + Search + Load + Session\&session id $=100642$ \&PHPSESSID=8ae013440a42242576057e 72718f4509.

3 Siehe dazu auch Beitrag auf Seite 2 und das neue ITA-Manu:script epub.oeaw.ac.att/ita/ita-manuscript/ita_09_02.pdf.
}

Ulrich Fiedeler, Michael Nentwich

\section{Mögliche Auswirkungen auf die Gesundheit durch künstlich hergestellte Nanomaterialien}

\begin{abstract}
Am 24. September fand die 3. NanoTrust-Herbsttagung statt. Während der Fokus der vorangegangenen Herbsttagungen auf Fragen der Risiko-Governance und der Regulierung von Nanotechnologien lag, befasste sich die diesjährige Konferenz mit toxikologischer Forschung zu Nanopartikeln und gab einen Überblick über aktuelle Forschungsergebnisse.
\end{abstract}

Noch vor wenigen Jahren bestimmten eher visionäre Themen wie Nanoroboter und „human enhancement" den Diskurs um die Nanotechnologie. Mittlerweile hat sich der Risikodiskurs fast ausschließlich auf mögliche Risiken von Nanopartikeln konzentriert. Unter den Fachleuten herrscht Einigkeit, dass negative Effekte, die mit der Nanotechnologie einhergehen könnten, am ehesten in diesem Bereich zu erwarten sind. Es gibt bereits eine Vielzahl von Forschungsaktivitäten, die sich den human- und ökotoxischen Wirkungen von Nanopartikeln widmen'. Auch wenn diese Forschung darauf abzielt, mögliche Risiken von Nanomateria- lien zu bewerten, so wirft sie derzeit mehr Fragen auf, als sie Antworten gibt. Vor diesem Hintergrund war es Ziel der Tagung, einen Überblick über die wesentlichen Forschungsfragen zu geben und mögliche Probleme zu identifizieren, die der Beantwortung dieser Fragen im Wege stehen.

Aus dem Vortrag von Georgios Katalagarianakis (EC, DG-Research) ging hervor, dass die EUKommission die Notwendigkeit einer Harmonisierung der nanotoxikologischen Forschungsaktivitäten erkannt hat. Daher fördert die EU im Bereich Risikoforschung besonders Projekte, welche sich mit der Zusammenführung von Wissen beschäftigen bzw. solche, die sich mit Instrumenten der Koordination und Kooperation von Forschungsprogrammen auseinandersetzen.

Wichtige Institutionen zur Identifikation neuer Risiken sind die "Scientific Committees on Emerging and Newly Identified Health Risks (SCENIHR), welche von der EU-Kommission zu verschiedenen Risikofragen einberufen werden. 
Mats-Olof Mattsson (SCENIHR) stellte die Arbeitsweise der Komitees vor und fasste ihre Empfehlungen zusammen. Als besonders dringliche Forschungsfragen wurden der Nachweis und die Charakterisierung von Nanopartikeln sowie deren Verhalten in lebenden Organismen genannt. Voraussetzung einer effektiven und belastbaren Forschung seien hierbei die Erstellung von einheitlichen Testverfahren sowie standardisierte Referenzmaterialien.

Hermann Stamm (JRC-IHCP) gab einen Überblick über die Vielzahl der verschiedenen Charakterisierungsmethoden von Nanomaterialien. Die Toxikologie könne sich auf ein umfangreiches Werkzeug zur Bestimmung der Eigenschaften von Nanopartikeln stützen. Er hielt fest, dass die Präparation des Untersuchungsmaterials einen entscheidenden Einfluss auf das Ergebnis der Messung habe.
Katja Nau (NanoCare, FZK) und Wolfgang Kreyling (Helmholtz Zentrum München) referierten über den derzeitigen Stand der nano-toxikologischen Forschung und stellten die aktuellsten Ergebnisse von In-vitro- und In-vivo-Studien vor.

Zusammenfassend lässt sich feststellen, dass Risikobewertung angesichts der Vielzahl von Aspekten bei der Herstellung, Charakterisierung und Untersuchung der toxikologischen Wirkungsmechanismen von Nanomaterialien nur mit einem "Case-by-case-Ansatz" sinnvoll stattfinden kann.

Das Programm sowie die Folien der Vorträge sind unter www.nanotrust.ac.at/nano09/ zu finden.

Aitken, Robert J., et al., 2009, EMERGNANO: A review of completed and near completed environment, health and safety research on nanomaterials and nanotechnology. Defra Project CB0409, Nr. TM/09/01, March 2009: Institute of Occupational Medicine (IOM)

www.safenano.org/Uploads/

EMERGNANO_CB0409_Full.pdf.

Ulrich Fiedeler

\section{Entzauberung der Nanotechnologie - Bericht von der ersten Konferenz der "Society for the Study of Nanoscience and Emerging Technologies"}

\begin{abstract}
Vom 8.-11. September fand gleich nach der offiziellen Gründung der "Society for the Study of Nanoscience and Emerging Technologies" (S.NET), deren erste Konferenz in Seattle statt. Ziel von S.NET ist es, den intellektuellen Austausch von Wissenschafterlnnen aus dem Bereich der Reflexionswissenschaften über die gesellschaftliche Bedeutung der Nanotechnologie zu fördern. Hauptaktivität von S.NET wird die Organisation jährlicher Konferenzen sein, die im Wechsel in Amerika und Europa stattfinden werden.
\end{abstract}

Nanotechnologie kann zum einen als Überbegriff von diversen Forschungsaktivitäten verstanden werden, deren Gemeinsamkeit in der Untersuchung und technologischen Anwendung von Strukturen in der Größenordnung von einem bis einige hundert Nanometern liegt. Auf der anderen Seite bezeichnet dieser Begriff aber auch die gesellschaftlichen Prozesse, die sich rund um diese Forschungsaktivitäten abspielen. Das beginnt bei der Forschungspolitik zur Nanotechnologie, geht über die Außendarstellung der Forscherlnnen sowie die Darstellung der Nanotechnologie in den Medien bis zur Reflexion der Transformationen des Wissenschaftsbetriebes. Die Konferenz im September hatte die nicht-technischen Aspekte der Nanotechnologie zum Thema.

Allein die Anzahl von insgesamt 120 Teilnehmerlnnen und etwa 80 Vorträgen zeigt das Interesse an der Analyse und Interpretation der $\mathrm{Na}$ notechnologie als gesellschaftliches Phänomen. Etwa zwei Drittel der TeilnehmerInnen kamen aus den USA, ein Drittel aus Europa, vorwiegend aus England und den Niederlanden.

In jeweils drei Parallelsessions wurde ein sehr breites Themenspektrum angeboten. Auch au- 
Berhalb des "Nanoethics Graduate Education Symposium" der University of Washington, das in die Tagung intergriert war, dominierten ethische Reflexionen zur Nanotechnologie die Veranstaltung. Der Plenar-Vortrag von René von Schomberg (EU-Kommission) widmete sich der Verantwortung der Wissenschafterlnnen. Weiters wurden konkrete ethische Aspekte medizinischer Anwendungen sowie das Thema Verteilungsgerechtigkeit behandelt. Weitere Vorträge beschäftigten sich mit analytischen Fragen wie der Destabilisierung von Orientierungsrahmen und symbolischer Ordnung oder mit Grenzüberschreitung von belebter und unbelebter Materie. Allgemein lässt sich feststellen, dass die Nanotechnologie neue Impulse bezüglich der Reflexion des Gegensatzes von Natur und Technik liefert.

Für den Autor war interessant zu beobachten, dass die philosophische Herangehensweise an das gesellschaftliche Phänomen der Nanotechnologie Zusammenhänge hervorhebt, die in den soziologischen oder politikwissenschaftlichen Analysen aus dem Blick geraten. So wurde beispielsweise in dem einleitenden Vortrag von Bernadette Bensaude-Vincent (Université Paris Ouest Nanterre) deutlich, dass sich Trans- formationen, die mit der Einführung der Nanotechnologie verbunden sind, auch in anderen wissenschaftlichen Bereichen wiederfinden lassen. Ein Beispiel dafür ist die Bedeutungsverschiebung, die sich aus dem Wechsel von der Erforschung der Materie zur Materialforschung vollzieht. Eine weitere wissenschaftliche Transformation deutet sich in der zunehmenden Auflösung der Trennung von Herstellung und Untersuchung der Struktur an. Beide Transformationen stehen in engem Zusammenhang mit dem Wandel in manchen Bereichen des Wissenschaftssystems, welche unter dem Begriff "Technoscience" diskutiert werden. Abgesehen von diesen eher erkenntnistheoretischen Fragen entzaubert der philosophische Blick durch das Aufdecken der geistigen Ursprünge die in der Nanotechnologie dominanten Denkmodelle, wie etwa den Traum von der Effizienzrevolution in der Produktion und der Dematerialisierung des Konsums. In dieser Hinsicht scheinen die philosophischen Beiträge die Diskussion um die Nanotechnologie "vom Kopf auf die Füße zu stellen" zu stellen.

Informationen zum S.NET sowie zum

Konferenz: www.thesnet.net.

Ulrich Fiedeler

\section{NANOtech Malaysia 2009 und Nanotechnologie-Forum in Kuala Lumpur}

\begin{abstract}
Vom 27. bis 29. Oktober fand in Kuala Lumpur die Konferenz NANOtech Malaysia statt. Auf Einladung der Organisatoren - unter ihnen die staatliche Universität (Universiti Kebangsaan Malaysia, UKM) und die Malaysische Akademie der Wissenschaften - hatte das ITA Gelegenheit, einen wesentlichen Konferenzbeitrag zu Sicherheits- und Risikothemen der Nanotechnologie zu gestalten.
\end{abstract}

Die NANOtech Malaysia fand seit Gründung des staatlichen Nanotechnologie-Forschungsprogramms (National Nanotechnology Initiative, NNI) im Jahre 2006 nun zum dritten Mal statt und stand im Zeichen der Etablierung der malaysischen Nanotechnologiegesellschaft (MNA). Die Eröffnung nahm der malaysische
Wissenschaftsminister. Im Rahmen der Hauptkonferenz hatten fünf österreichische Delegierte die Gelegenheit, eingeladene Vorträge zu halten:

André Gazsó (ITA) gab in seinem Plenarvortrag eine Übersicht über die aktuelle europäische Situation der Risikoregulierung der $\mathrm{Na}$ notechnologien und stellte das Projekt NanoTrust, seine Aufgaben und Produkte vor. Myrtill Simkó (ITA) referierte über den aktuellen Stand der humantoxikologischen Forschung zu synthetischen Nanopartikeln und deren Interaktion mit lebender Materie. Mats-Olof Mattsson (Universität Wien/SCENIHR) gab eine fundierte Einführung in die Arbeit des SCENIHR, des wissenschaftlichen Rates der EU-Kommission zu Fragen neuartiger Gesundheitsrisiken (Scientific Committee on Emerging and Newly Iden- 
tified Health Risks. Anschließend wurde über die Ergebnisse der bisher vier Veröffentlichungen von SCENIHR zum Thema Nanotechnologie diskutiert. Andreas Falk (BioNanoNet) berichtete von österreichischen Forschungen zur Nanotoxikologie, die im Rahmen seines Forschungsverbundes durchgeführt werden. Alexander Pogany (BMVIT) stellte schließlich die Situation der Forschungspolitik im Bereich Nanotechnologie in Europa und in Österreich dar, wobei neben der Beschreibung der Technologieförderung die Aktivitäten im Bereich Sicherheitsund Risikoforschung einen speziellen Schwerpunkt bildeten.

André Gazsó war weiters Gastredner auf dem parallel stattfindenden Nanotechnologie-Forum („Nanotechnology: Evolution of Knowledge to Market"), das von der malaysische Akademie der Wissenschaften am 28. Oktober organisiert wurde. Als einziger Europäer diskutierte er mit Kollegen aus Indien, Taiwan und Südkorea zu Fragen des nachhaltigen und verantwortungsvollen Einsatzes innovativer Ent- wicklungen am Beispiel der Nanotechnologien. Die Teilnehmerlnnen aus Österreich wurden schließlich für den 29. Oktober eingeladen, in einem vom Institut für Medizinische Mikrobiologie (UMBI) der Universität Kuala Lumpur veranstalteten Seminar über Nanotechnologien und Gesundheit vor Fachpublikum zu sprechen.

Durch die Teilnahme eine starken österreichischen Delegation bei dieser wichtigen asiatischen Konferenz konnten nicht nur wesentliche Themen der Risikoforschung in diesem Rahmen eingebracht werden, sondern auch zahlreiche weiterführende Kontakte geknüpft bzw. erneuert werden, die auch der österreichischen Nano- und Nanorisikoforschung zugute kommen werden.

Weitere Informationen:

www.nanotechmalaysia.com.my, www.umbi.ukm.my/umbi/index.php?option= com content\&task $=$ view\&id $=218 \&$ Itemid $=56$.

André Gazsó

\section{Aktuelle Publikationen}

\section{Referierte Artikel}

Aichholzer, G., Allhutter, D., 2009, Public Policies on eParticipation in Austria, in: A. Macintosh, E. Tambouris (eds.), Electronic Participation, First International Conference, ePart 2009, Linz, Austria, September, 2009, Proceedings, Lecture Notes in Computer Science LNCS 5694, Berlin, Heidelberg, New York: Springer, 24-35.

Aichholzer, G., Strauß, S., 2009, The Citizen's Role in National Electronic Identity Management. A Case-study on Austria, in: Dini, O., Hoffmann, M., Klyuev, V., Noll, J., Su, W. (eds.), The Second International Conference on Advances in Human-Oriented and Personalized Mechanisms, Technologies, and Services CENTRIC 2009, 20-25 September 2009, Porto, Portugal, IEEE Computer Society, 45-50.

Mild, K.H., Wilén, J., Mattsson, M.O., Simkó, M., 2009, Background magnetic fields in incubators: A factor of importance in cell culture work, Cell Biology International, Vol. 33/Issue 7 (July 2009), pp. 755 ff.

[http://www.sciencedirect.com/science?_ob= PublicationURL\&_tockey $=\% 23 \mathrm{TOC} \% 23$ $6734 \% 232009 \% 23999669992 \% 23$ $1239086 \% 23 F L A \% 23 \&$ cdi $=6734 \&$ pubType $=\mathrm{J} \&$ auth $=\mathrm{y} \&$ acct $=\mathrm{C} 000050221 \&$ version $=1 \&$ urlVersion $=0 \&$ userid $=10 \& \operatorname{md} 5$ $=9 \mathrm{e} 8484 \mathrm{~d} 873 \mathrm{cbbdf} 7 \mathrm{f} 3519 \mathrm{e} 3 \mathrm{~d} 8 \mathrm{c} 587119]$.

Schmidt,M., Ganguli-Mitra,A., Torgersen, H., Kelle, A., Deplazes, A., Biller-Andorno, N., 2009, A priority paper for the societal and ethical aspects of synthetic biology, Systems and Synthetic Biology, vol. 3, nrs. 1-4, pp. 3-7, doi 10.1007/s1 1693-009-9034-7. 
Torgersen, H., 2009, Synthetic Biology in Society - Learning from past Experience? Systems and Synthetic Biology, Vol 3, nos. 1-4, pp. 9-17, doi 10.1007/s1 1693-009-9030-y.

\section{Artikel/Buchbeiträge}

Aichholzer, G., Kozeluh, U., 2009, Evaluating e-Democracy, in: EuroSpace Group (ed.), eGov 2.0: pave the way for e-participation, Rome: EuroSpace Group, 50-55

[http://www.eurospacegroup.com/img/pubblic azioni/volume/volume_2009.pdf].

Aichholzer, G., 2009, The Delphi Method: Eliciting Experts' Knowledge in Technology Foresight, in: A. Bogner, B. Littig, W. Menz (eds.), Interviewing Experts, Basingstoke: Palgrave Macmillan, 252-273.

Allhutter, D. (2009). Software Design: a deconstructivist feminist approach. Position paper for the European Conference on Computer Supported Cooperative Work (ECSCW'09), Workshop 'Considering Gender in $\mathrm{ECSCW}^{\prime}, 8^{\text {th }}$ of September, Vienna. [http://www.ics.uci.edu/ jen/Papers/ Allhutter.pdf].

Bogner, A., Kastenhofer, K. und Torgersen, H., 2009, Inter- und Transdisziplinarität - zur Einleitung in eine anhaltend aktuelle Debatte, in: Bogner, A., Kastenhofer, K. und Torgersen, H. (Hg.): Inter- und Transdisziplinarität im Wandel? Neue Perspektiven auf problemorientierte Forschung und Politikberatung, Baden-Baden: nomos, 7-21.

Bogner, A., 2009, Mikropolitik des Wissens. Macht und Geltung in interdisziplinären Gremien, in: Bogner, A., Kastenhofer, K. und Torgersen, $\mathrm{H}$. (Hg.): Inter- und Transdisziplinarität im Wandel? Neue Perspektiven auf problemorientierte Forschung und Politikberatung, Baden-Baden: nomos, 205 228.

Bogner, A., Kastenhofer, K., 2009, Biopolitik, in: Soziologische Revue 32(4), 391-400.
Wächter, P., 2009, Scenarios for a Sustainable Energy Supply: Results of a Case Study for Austria: in Ardil, C., (ed.), World Academy of Science, Engineering and Technology, Conference proceedings, International Conference on Energy, Environment and Sustainable Development 2009, ISSN 20703724, Paris 2009, pp.315-322.

Fiedeler, U. und Nentwich, M., 2009, Begleitforschung. Zur Klärung eines politischen Begriffs, Technikfolgenabschätzung - Theorie und Praxis 18(2), 94-102

[http://www.itas.fzk.de/tatup/092/fine09a.pdf].

Kastenhofer, K., 2009, Zur Gegenstandsbestimmung einer Soziologie des Nichtwissens bei Wehling, Erwägen - Wissen - Ethik 20(1), 135-138.

Kastenhofer, K., 2009, Zwischen "schwacher" und "starker" Interdisziplinarität: Sicherheitsforschung zu neuen Technologien, in: Bogner, A., Kastenhofer, K. und Torgersen, $\mathrm{H}$. (Hg.): Inter- und Transdisziplinarität im Wandel? Neue Perspektiven auf problemorientierte Forschung und Politikberatung, Baden-Baden: nomos, 87-122.

Nentwich, M., 2009, Cyberscience 2.0 oder 1.2? Das Web 2.0 und die Zukunft der Wissenschaft. ITA manu:scripts, Nr. ITA-09-02 hrsg. v. Institut für Technikfolgen-Abschätzung [http://epub.oeaw.ac.at/ita/ ita-manuscript/ita_09_02.pdf].

Sterbik-Lamina, J., Peissl, W., 2009, Zertifizierter Datenschutz in Europa möglich - Ergebnisse aus dem Projekt EuroPriSe, in: Technikfolgenabschätzung - Theorie und Praxis Nr. 3, 18. Jg, Dezember 2009.

Nentwich, M., 2009, Zitatenindices als Zerrspiegel und Herausforderung, GAIA (4), 281-283.

Sterbik-Lamina, J., 2009, Wie vereint man den Schutz kritischer Infrastrukturen mit dem Schutz der Privatsphäre?, in: Seböck, W. und Huber, E. (Hrsg.), Tagungsband der 7. Information Security Konferenz 2009 - DonauUniversität Krems 29.10.2009, Wien: Österreichische Computer Gesellschaft books@ocg.at, Band 257, S. 29-41. 
Suschek-Berger, J., Ornetzeder, M., 2009, Kooperative Sanierung. Einbeziehung von BewohnerInnen bei nachhaltigen Sanierungsprozessen in Geschoßwohnbauten, in: Ökosan'09, Internationales Symposium für hochwertige energetische Sanierung von großvolumigen Gebäuden, Tagungsband, Institut für Nachhaltige Technologien, Gleisdorf, S. 89-96.

\section{Bücher/Herausgeberschaften}

Bogner, A., Kastenhofer, K. und Torgersen, $\mathrm{H}$. (Hg.), 2009, Inter- und Transdisziplinarität im Wandel? Neue Perspektiven auf problemorientierte Forschung und Politikberatung; in Reihe: Wissenschafts- und Technikforschung, hg. v. Bora, A., Maasen, S., Reinhardt, C. und Wehling, P., Baden-Baden: nomos.

\section{Konferenzbeiträge/Vorträge}

Aichholzer, G., Strauß, S., 2009, The Austrian Case: Multi-card concept and the relationship between citizen ID and social security cards, Workshop 1: Introducing elDs as a Multi-Facet Innovation, Eighth International EGOV Conference 2009 within the DEXA conference cluster, August 30 - September 3, 2009, Linz, Austria.

Aichholzer, G., Allhutter, D., 2009, Public Policies on eParticipation in Austria, First International Conference on eParticipation (ePart) 2009 within the DEXA conference cluster, September 1-3, 2009, Linz, Austria.

Aichholzer, G., Strauß, S., 2009, The Citizen's Role in National Electronic Identity Management. A Case-study on Austria, The Second International Conference on Advances in Human-Oriented and Personalized Mechanisms, Technologies, and Services CENTRIC 2009, 20-25 September 2009, Porto, Portugal.

Aichholzer, G., Strauß, S., 2009, Bürgerbeteiligung bei Energiesparmassnahmen - Transparente Selbstverpflichtung zum Klimaschutz, Internationales Vergleichsprojekt "e2democracy", Bregenz, 6.1 1.2009.

\section{Forschungsberichte}

Simkó, M., Fiedeler, U., Gazsó, A. und Nentwich, M., 2009, Können Nanopartikel in das Gehirn gelangen? NanoTrust-Dossiers, Nr. 014 hrsg. v. Institut für TechnikfolgenAbschätzung (ITA), Wien: ITA

[http://epub.oeaw.ac.at/ita/ nanotrust-dossiers/dossier014.pdf].

Bogner, A., 2009, Wissenschafts- und Technikkontroversen, Vortrag auf dem Kongress der Österreichischen Gesellschaft für Soziologie, 24.-26.09., Graz.

Bröchler, S., Sotoudeh, M., Decker, M. und NTA Koordinationsteam, 2009, 1. Online-Mitglieder-Befragung zum Netzwerk Technikfolgenabschätzung (NTA), Erste Ergebnisse, NTA Jahrestreffen, 11. November, Berlin.

Čas, J., 2008, Ethical aspects of Security Research - Privacy and Freedom enhancing technologies, ESRIF WG 11 meeting, $4^{\text {th }}$ September, Brussels

Fiedeler, U., 2009, Vision Assessment of Nanotechnology - The role of visions in research programs, S.Net Conference 2009, September 8-11, Washington State University, Seattle.

Fiedeler, U., 2009, Preliminary Considerations on a Risk Assessment of Converging Technologies, STP 2009, Converging Technologies, Changing Societies, 08.07.2009, Washington State University, Seattle. 
Gazsó, A., 2009, Nanotechnologie: Risiko und Natürlichkeit. Eingeladener VOrtrag bei der "SIZE MATTERS 2009: Ethische Herausforderungen der Nanotechnologie", Saarbrücken, 17. Juni 2009.

Gazsó, A., 2009, Nanomaterial: Risk, Health and Safety. Invited lecture and panelist at the "Forum "Nanotechnology: Evolution of Knowledge to Market", Academy of Science Malaysia, October 28, 2009.

Gazsó A., Risk Governance of Nanotechnologies. Invited pleanry lecture at the NANOtech Malaysia 2009, Kuala Lumpur Convention Centre, October 28, 2009.

Gazsó, A., Risk Governance of Nanotechnologies - The Austrian Project "NanoTrust". Invited lecture at the "Seminar on Nanotechnology in Health and Medicine, National University of Malaysia", Kuala Lumpur, UMBI (Medical Molecular Biology Institute), October 29, 2009.

Gazsó, A., Das Projekt NanoTrust - Ein Beitrag zur Risikoregulierung der Nanotechnologien in Österreich. Eingeladener Vortrag am 3. internationalen Behördendialog, Wien, Lebensministerium, 10. November 2009.

Gazsó, A., 2009, Der gesellschaftliche Umgang mit neven Technologien am Beispiel der Nanotechnologie. BG/BRG Gleisdorf, 11. November 2009.

Gazsó, A., 2009, Nanotechnologie und Vorsorgeprinzip - Der gesellschaftliche Umgangmit neven Technologien. Eingeladener Vortrag auf der Tagung "Nanotechnologie im Lichte der aktuellen Diskussion zu REACH und CLP", Wirtschaftskammer Österreich, 12. November 2009.

Kastenhofer, K., 2009, Wissenschaftskulturen in den Naturwissenschaften: Der Umgang mit Nichtwissen und Risiko, Forschungstag "Wissenschaftskulturen", 11.11.2009, Universität Klagenfurt.

Kastenhofer, K., 2009, Beyond science: Integration of science, civil society and politics in parliamentary debates on medical biotechnology regulation in the UK, Integration in Inter- and Transdisciplinary Research. Forging Collective Concepts, Methods and Practices - Changing Structures, 19-21 November, Universität Bern.
Lantow, M., Mattsson, M.O., Weiss, D.G., Simkó, M., 2009, Responding and non-responding human Mono Mac 6 cell line cells to electromagnetic field exposure, BioEM 2009 - Joint Meeting of The BEMS, 16.06.2009, Davos $(\mathrm{CH})$.

Nentwich, M., 2009, Statement am Podium "Wirkung von Nanotechnologie. Wirtschaftlicher Fortschritt und neve Märkte im Spannungsfeld der Technologiefolgenabschätzung", Nano. Wissenschaft. Wirtschaft. Wirkung 09 (NANO Initiative), 9.11., Techgate Wien.

Nentwich, M., 2009, Cyberscience 1.2: Potenziale des Web 2.0 für das Netzwerk TA, 5. Jahrestreffen des Netzwerks Technikfolgenabschätzung (NTA), 12.11., Berlin, Bundestag [http://www.oeaw.ac.at/ita/ebene5/ Cyberscience_2.0or1.2-NTA.pdf].

Ornetzeder, M. und Suschek-Berger, J., 2009, Sanierung und Partizipation - Kooperation oder Konflikt? Ökosan'09, Internationales Symposium für hochwertige energetische Sanierung von großvolumigen Gebäuden, 08.10., Weiz, Österreich

[http://www.aee-intec.at/index.php? seitenName $=$ veranstaltungenDetail\& veranstaltungenId=174]

Ornetzeder, M., 2009, Sustainable technology and the critical user: Experiences with focus group discussions between research and participation, Second German Environmental Sociology Summit, 06.11., Leipzig, Germany.

Peissl, W., 2009, Privacy and Security - a way to manage the dilemma, Information Security Solutions European Conference ISSE 2009, 6. - 8.10. The Hague.

Peissl, W., 2009, Privacy and Security, Results from the PRISE project, Privacy OS $27^{\text {th }}$ October, Vienna.

Peissl, W., 2009, Self-Regulation \& Privacy: The Austrian Case, Privacy OS $27^{\text {th }}$ October, Vienna.

Simkó, M., 2009, Nutzen und Risiken synthetischer Nanopartikel, Ringvorlesung Tycho Net, Universität Rostock, 07.07.2009, Rostock (D). 
Simkó, M., 2009, Nanoparticles: Biological Effects and Approaches to Risk Assessment, Materials' Days, University of Rostock, 09. 07.2009

Sterbik-Lamina, J., 2009, Sicherheit versus Privatsphäre? - Wie vereint man den Schutz Kritischer Infrastrukturen mit dem Schutz der Privatsphäre?, Information Security Konferenz 2009, 29.10.2009, Donau-Universität Krems.

Torgersen, H., 2009, From Risk Assessment to Decision Making, OECD Roundtable on Risk Governance Policy for Nanotechnology, September 25th, Vienna, Austria.

\section{Sonstiges/Kurzbeiträge}

Wächter, P., 2009, Sustainability and Communities of Place, Book Review, Ed: Carl A. Maida. In: Environmental Politics, Volume 18, Number 4

Impressum:

Medieninhaber:

Österreichische Akademie der Wissenschaften

Juristische Person öffentlichen Rechts (BGBI 569/1921

idF BGBI I 130/2003)

Dr. Ignaz Seipel-Platz 2, A-1010 Wien

Herausgeber:

(C) Institut für Technikfolgen-Abschätzung (ITA)

Strohgasse 45/5, A-1030 Wien

Alle Rechte vorbehalten.
Torgersen, H., 2009, Genetically modified Plants and Food - challenges and future issues in Europe, First European Innovation Summit, 13-14.10.2009, European Parliament, Brussels.

Torgersen, H., 2009, Jenseits der Gentechnik - Systembiologie, Synthetische Biologie und 'embedded TA'. Kolloquium im Rahmen des DFG Graduiertenkollegs "Auf dem Weg in die Wissensgesellschaft", 19.10.2009, Univ. Bielefeld.

Torgersen, H., 2009, Synthetische Biologie Wein in welchen Schläuchen? Soziale Technik 3/2009, S. 3-6.

Der ITA-Newsletter erscheint viermal jährlich und enthält Informationen über nationale und internationale Trends in der Technikfolgen-Abschätzung, ITA-Forschungsprojekte, Publikationen der ITA-MitarbeiterInnen und ITA-Veranstaltungen. Das ITA verfolgt mit diesem periodischen Medium das Ziel, wissenschaftliche Zusammenhänge zwischen Technik und Gesellschaft einem breiten Leserlnnenkreis zugänglich zu machen. 


\section{Aktuelle Buchpublikationen des ITA}

Inter- und Transdisziplinarität im Wandel? Neue Perspektiven auf problemorientierte Forschung und Politikberatung.

Bogner, A., Kastenhofer, K. und Torgersen, H. (Hg.) in Reihe: Wissenschafts- und Technikforschung, hg. v. Bora, A., Maasen, S., Reinhardt, C. und Wehling, P., 2009, Baden-Baden: nomos.

Interviewing Experts.

Bogner, A., Littig, B., Menz, W. (Hrsg.)

2009, Palgrave Macmillan, Basingstoke

Experteninterviews. Theorien, Methoden, Anwendungsfelder.

Bogner, A., Littig, B., Menz, W. (Hrsg.), 3. grundlegend überarb. Aufl. 2009, Verlag für Sozialwissenschaften, Wiesbaden

Technical education for sustainability. An analysis of needs in the $21^{\text {st }}$ century.

Sotoudeh, M., in Reihe: Environmental Education, Communication and Sustainability, Bd. 30, hg. v. Filho, W. L.,

2009, Peter Lang Internationaler Verlag der Wissenschaften, Frankfurt am Main

Das ÖAW-Institut für Technikfolgen-Abschätzung.

Nentwich, M. (Hg.)

2008, Institut für Technikfolgen-Abschätzung, Eigenverlag

Europäische Öffentlichkeit und medialer Wandel. Eine transdisziplinäre Perspektive. Wolfgang R. Langenbucher, Michael Latzer ( $\mathrm{Hg}$.)

2006, Verlag für Sozialwissenschaften: Wiesbaden

Handbuch Strategische Umweltprüfung - Die Umweltprüfung von Politiken, Plänen und Programmen. 4. Ergänzungslieferung Mai 2006

ITA/Michael Nentwich (Hg.), Kerstin Arbter (Bearbeiterin)

Verlag der Österreichischen Akademie der Wissenschaften: Wien

Leiffaden Partizipativer Verfahren. Ein Handbuch für die Praxis.

Steyaert, S., Lisoir, H., Nentwich, M. (Hg.)

2006, Flemish Institute for Science and Technology Assessment, König-Baudouin-Stiftung, Institut für Technikfolgen-Abschätzung:

Brüssel/Wien

Nähere Informationen unter: www.oeaw.ac.at/ita/books.htm 

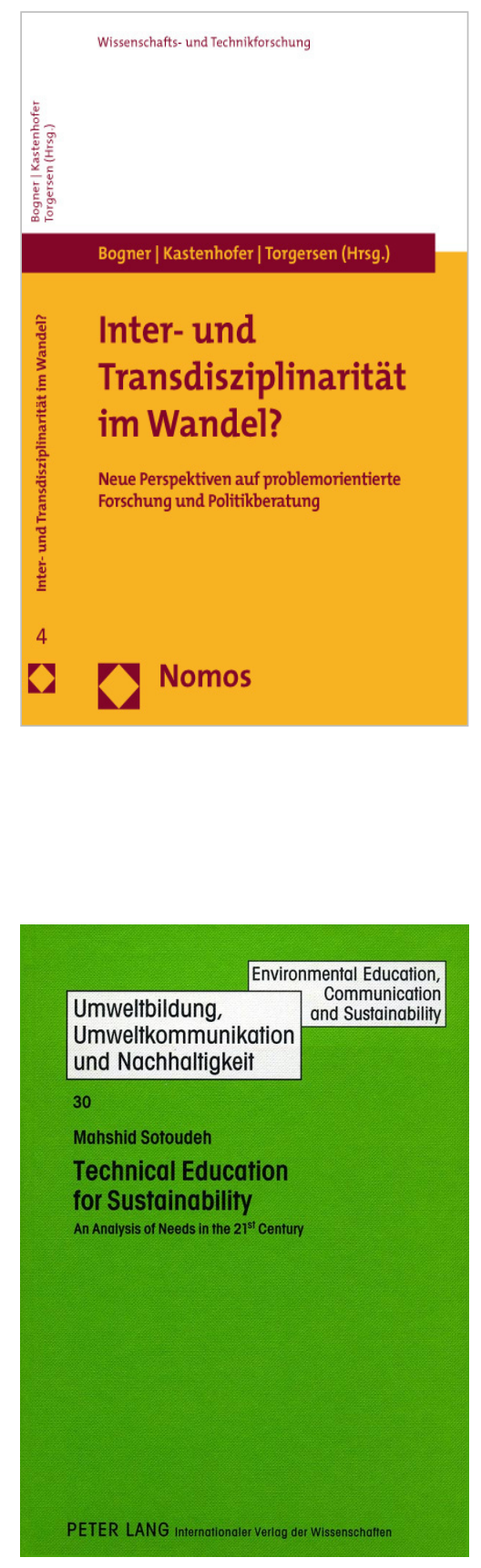

\section{Sotoudeh, M., 2009, Technical education for sustainability. An analysis of needs in the $21^{\text {st }}$ century. Erschienen in der Reihe: Environmental Education, Communication and Sustainability, Frankfurt/Main: Verlag Peter Lang}

Inhalt des Bandes ist die Analyse des Themas "Technische Ausbildung und Nachhaltigkeit" aus drei unterschiedlichen Perspektiven:

Teil I (Engineering) handelt von den verschiedenen Rollen technischer Berufe in der Gesellschaft, unter anderem auf Basis der Ergebnisse einer Recherche und Befragung aus dem Jahr 2006.

Teil II (Technology - Engineering) zeigt den Kontext der technischen Berufe im Rahmen einer nachhaltigen Entwicklung auf. Der Schwerpunkt der Ausführungen liegt auf Technikfolgenabschätzung und deren Bedeutung für technische Berufe.

Teil III (Technical education) bietet eine Zusammenschau wichtiger expliziter Anforderungen an (technische) Universitäten und diskutiert - basierend auf den Teilen I \& II - einige grundsätzliche Ziele für technische Universitäten. In diesem Teil werden zwei Empfehlungen für die Begleitung der Reformen an technischen Universitäten durch partizipative Verfahren entwickelt. Unter Beachtung der Autonomie der Universitäten sollten gesellschaftlichen Anforderungen berücksichtigt werden.

Das Buch ist ein Beitrag zu den Debatten im Rahmen der UNDekade "Bildung für nachhaltige Entwicklung" (2005-2014). 
ITA-Seminar: 11. Jänner 2010, 16:00-18:00 Uhr

Dr. Markus Schmidt

Organisation for International Dialogue and Conflict Management (IDC)

\section{„Safety and ethical aspects of synthetic biology"}

Ort: Bibliothek des ITA, 1030 Wien, Strohgasse 45, 3. Stock, Tür 5

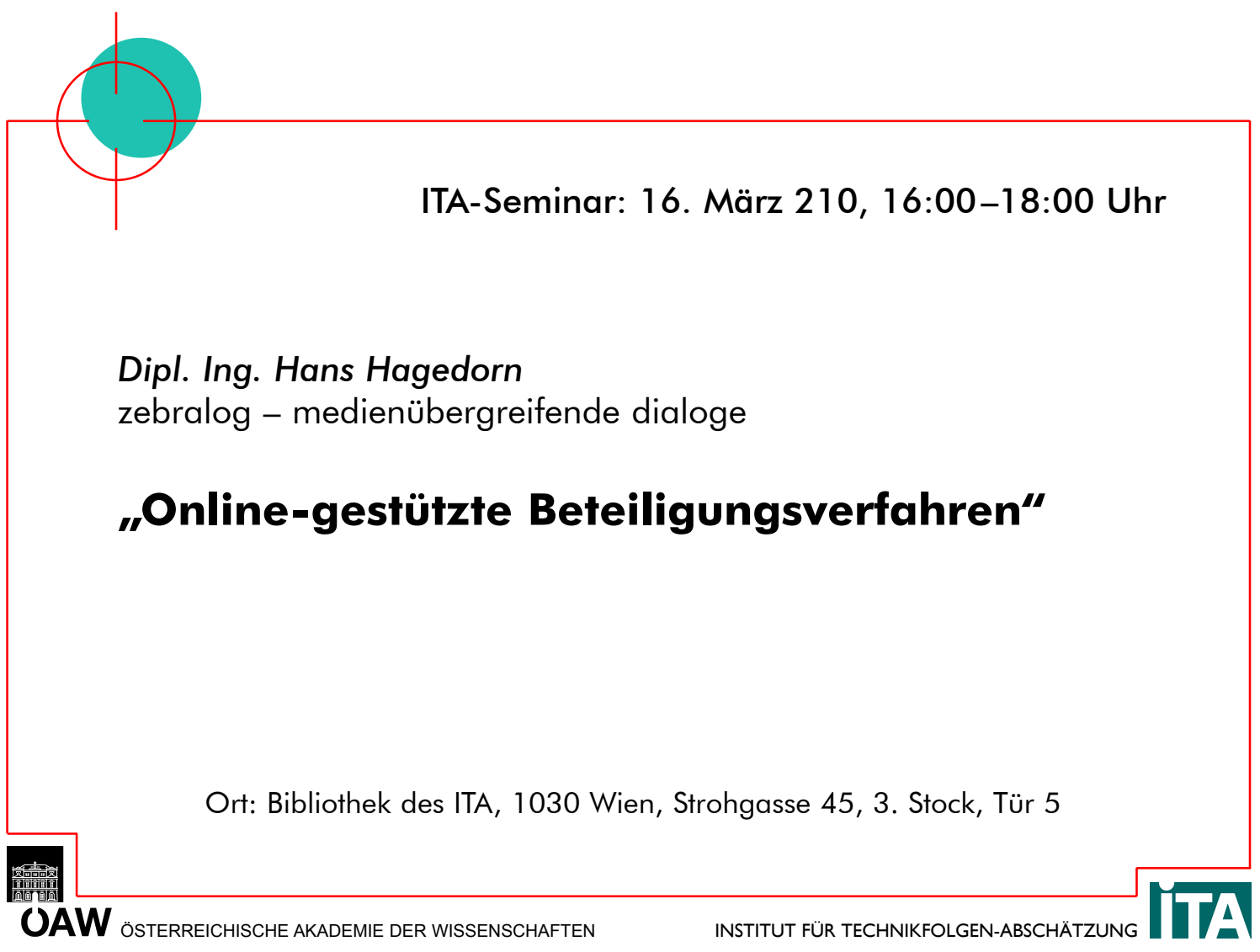


Konferenz: 16.-17. April 2010, 12:00-15:00 Uhr

\section{GAIA-Jahrestagung 2010}

Österreichische Akademie der Wissenschaften

A-1010 Wien, Dr.-Ignaz-Seipel-Platz 2

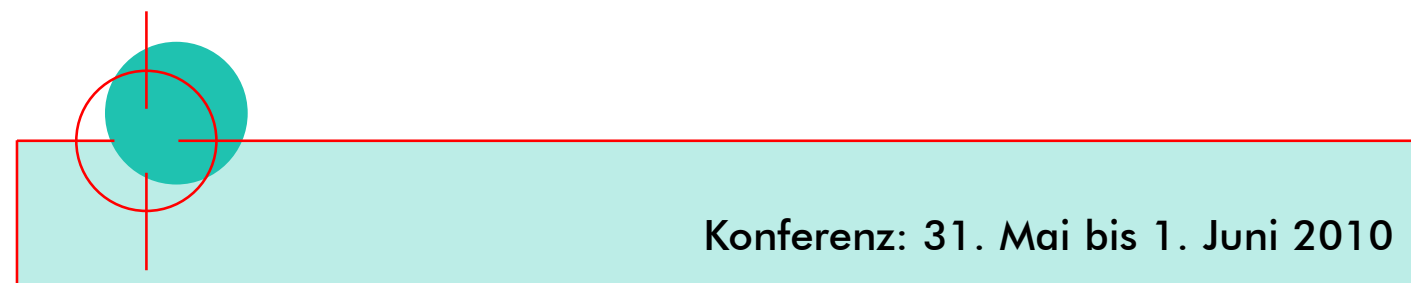

\section{TA'10}

Wann TA?

Die Ethisierung der Technik - und ihre Bedeutung für die Technikfolgenabschätzung

Die 10. internationale Technikfolgenabschätzungskonferenz in Wien Österreichische Akademie der Wissenschaften A-1010 Wien, Dr.-Ignaz-Seipel-Platz 2, www.oeaw.ac.at/ita/ta10/ 
Die 14 News werden herausgegeben vom Institut für Technikfolgen-Abschätzung der Österreichischen Akademie der Wissenschaften (ITA). Für weiterführende Fragen zu den in dieser Ausgabe behandelten Themen und zur Technikfolgen-Abschätzung im Allgemeinen stehen wir lhnen gerne zur Verfügung:

Institut für Technikfolgen-Abschätzung (ITA) der Österreichischen Akademie der Wissenschaften

A-1030 Wien, Strohgasse 45/5/3. Stock

Tel.: +43-1-51581/6582, Fax: +43-1-710 9883

E-Mail: tamail@oeaw.ac.at, www.oeaw.ac.at/ita

Leiter des Instituts:

Univ.-Doz. Mag. Dr. Michael NENTWICH...DW 6583.....mnent@oeaw.ac.at

\section{MitarbeiterInnen:}

Mag. Dr. Georg AICHHOLZER

.DW6591.....aich@oeaw.ac.at

Mag. ${ }^{a}$ Dr. ${ }^{\text {in }}$ Doris ALLHUTTER

DW 6585.....dallhutt@oeaw.ac.at

Mag. ${ }^{a}$ Dr. ${ }^{\text {in }}$ Ulrike BECHTOLD

.DW 6593.....ubecht@oeaw.ac.at

Dipl.-Soz. Dr. Alexander BOGNER

.DW 6595.....abogner@oeaw.ac.at

Ing. Mag. Johann ČAS

.DW6581....jcas@oeaw.ac.at

Dipl.-Phys. Dr. Ulrich FIEDELER

DW6577.....ufiedeler@oeaw.ac.at

MMag. Dr. André GAZSÓ

.DW 6578.....agazso@oeaw.ac.at

Mag.(FH) Werner KABELKA

DW 6587.....kabelka@oeaw.ac.at

Mag. a Dr. ${ }^{\text {in }}$ Karen KASTENHOFER

DW 6580.....kkast@oeaw.ac.at

Mag. Dr. Michael ORNETZEDER.

DW6589.....ornetz@oeaw.ac.at

Mag. Dr. Walter PEISSL

DW 6584.....wpeissl@oeaw.ac.at

Barbara POPPEN.

.DW 6582.....bpoppen@oeaw.ac.at

Prof. in Dr. ${ }^{\text {in }}$ Myrtill SIMKÓ

DW 6579.....msimko@oeaw.ac.at

DI ${ }^{\text {in }}$ Dr. ${ }^{\text {in }}$ Mahshid SOTOUDEH

DW 6590.....msotoud@oeaw.ac.at

Sabine STEMBERGER

DW 6586.....sstem@oeaw.ac.at

Jaro STERBIK-LAMINA, M.Sc

.DW 6594.....jsterbik@oeaw.ac.at

Mag. Stefan STRAUSS

.DW 6599.....sstrauss@oeaw.ac.at

Dr. Helge TORGERSEN

.DW 6588.....torg@oeaw.ac.at

Mag. ${ }^{a}$ Petra WÄCHTER

.DW 6592.....pwaecht@oeaw.ac.at

E-Mail-Newsservice: Wenn Sie an Berichten, Newslettern, Veranstaltungshinweisen etc. interessiert sind, registrieren Sie sich bitte unter https://lists.oeaw.ac.at/mailman/listinfo/itanews. 